\title{
¿Qué lugar ocupa la cultura en el Presupuesto Nacional? Un breve análisis descriptivo de la evolución de la asignación presupuestal en cultura 1999-2018
}

\author{
The place of culture in the National Budget. A short descriptive \\ analysis of the evolution of national budget allocation to culture, \\ 1999-2018
}

\section{Hernán Cabrera*}

\footnotetext{
* Licenciado en Sociología y especialista en Gestión Cultural, Facultad de Ciencias Sociales, Universidad de la República, Uruguay. Maestrando en Cultura Pública por el Instituto de Cultura Pública del Ministerio de Cultura de la Nación de la República Argentina. Responsable del Sector Cultura en la Dirección de Planificación de la Oficina de Planeamiento y Presupuesto, Uruguay. $\triangle$ hernan.cultura@gmail.com
}

RECIBIDO: [1.4.2018] ACEPTADO: [9.5.2018]

\begin{abstract}
Resumen
El propósito de este artículo es indagar sobre la participación de la cultura en el Presupuesto Nacional mediante un análisis descriptivo de la evolución de la asignación y su ejecución en los últimos dos decenios. Pese al crecimiento sostenido de la economía uruguaya durante los últimos quince años, se verá que la asignación presupuestal es muy marginal. Además podrán identificarse algunos cambios en la institucionalidad que se reflejan significativamente en la evolución de la asignación del presupuesto en cultura. Al mismo tiempo, podrá verse un cambio, iniciado en el 2005 e intensificado en el 2015, que muestra una desconcentración de la gestión presupuestal en cultura desde la sede del Ministerio de Educación y Cultura hacia el resto de las unidades ejecutoras que forman parte del núcleo ejecutor de las políticas públicas en cultura. Finalmente, se muestran los datos de ejecución presupuestal y se hace una pequeña digresión teórica respecto a ella que propone la necesidad de repensar las formas en que se ven las políticas públicas en cultura, tanto por los decisores presupuestales como por los gestores culturales.
\end{abstract}


Palabras clave: economía cultural, industria cultural, gestión cultural, presupuesto cultural, Uruguay.

\begin{abstract}
This paper studies the budget expenditure devoted to culture, through a descriptive analysis of the evolution of national budget allocation and execution during the last twenty years. Despite the sustained growth of Uruguayan economy during the last fifteen years, it will be seen that budget allocation to culture is very marginal. In addition, changes in the institutional framework, significantly reflected in the evolution of budget allocation in culture, can be identified. At the same time, deconcentration of budget management from the Ministry of Education and Culture's head office to the rest of the Executing Units operating at the core of culture public policies is evidenced, throughout a process initiated in 2005 and intensified in 2015. Finally, a small theoretical digression is made according to the budget execution data shown, pointing towards the need to rethink the ways in which public cultural policies are viewed, either by budget decision-makers or by cultural managers.
\end{abstract}

Keywords: economics of culture, cultural industry, cultural management, cultural budgets, Uruguay.

\title{
Introducción
}

Este trabajo tiene por objeto indagar en torno a los cambios que se presentan en la asignación y ejecución del Presupuesto Nacional (PN) para el funcionamiento, la inversión y el pago de personal de las instituciones públicas que tienen tareas específicamente dedicadas a la cultura y que se encuentran en la órbita del Ministerio de Educación y Cultura (MEC), es decir, las unidades ejecutoras (UE) especializadas en la materia.

Para ello se trabaja con la línea temporal 1999-2018, por dos grandes motivos. Por un lado, los datos que se encuentran disponibles para años previos a 1999 no cuentan con los niveles de desagregación necesarios para investigar con la suficiente profundidad la participación de las UE en la asignación y ejecución del PN. Por otro lado, este corte temporal permite ver - aunque no es un tema en el que se profundizará- la evolución y los cambios presupuestales de los últimos 20 años, ejercicio interesante por cuanto abarca el último período del Partido Colorado en el Poder Ejecutivo, una de las crisis económicas más fuertes del Uruguay, y los tres períodos del Frente Amplio en el poder.

Este artículo busca efectuar un análisis descriptivo de las asignaciones y ejecuciones presupuestales, sus cambios y permanencias en ese período, por lo que no pre- 
tende una gran densidad analítica, sino que busca abrir puertas a nuevas investigaciones que permitan avanzar en estos temas que parecen tan ajenos al quehacer de la gestión cultural.

\section{De las definiciones. La asignación de presupuesto en cultura en el Presupuesto Nacional}

Lo que se pretende en esta primera sección es formular algunas definiciones que den marco al resto del trabajo que aquí se presenta. Posiblemente, una de las primeras interrogantes que surgen es qué se considerará cultura, ya que sin esa definición todas las enunciaciones que vengan después serán más vulnerables a cuestionamientos. Ya que lo que se pretende en este trabajo es echar luz sobre qué lugar ocupa la cultura en el PN, se tomará la definición de cultura de la Declaración de la UNESCO sobre las Políticas Culturales (aprobada en México en 1982), donde se la entiende como:

[...] el conjunto de los rasgos distintivos, espirituales y materiales, intelectuales y afectivos que caracterizan a una sociedad o un grupo social. Ella engloba, además de las artes y las letras, los modos de vida, los derechos fundamentales al ser humano, los sistemas de valores, las tradiciones y las creencias.

La Declaración agrega:

La cultura da al hombre la capacidad de reflexionar sobre sí mismo. Es ella la que hace de nosotros seres específicamente humanos, racionales, críticos y éticamente comprometidos. A través de ella discernimos los valores y efectuamos opciones. A través de ella el hombre se expresa, toma conciencia de sí mismo, se reconoce como un proyecto inacabado, pone en cuestión sus propias realizaciones, busca incansablemente nuevas significaciones, y crea obras que lo trascienden.

Si bien es una definición muy general y difícil de operacionalizar en términos presupuestales — tarea que no será desarrollada en este trabajo-, se la elige por ser producto de un acuerdo entre los Estados miembros del mencionado organismo internacional, al tiempo que ha sido referente para todos ellos en posteriores declaraciones o convenciones, así como para las legislaciones nacionales de muchos, entre los que se encuentra Uruguay.

Siguiendo esta línea de pensamiento, se requieren algunas definiciones adicionales que permitan comprender el encuadre de este trabajo. La primera es la de políticas públicas, que serán entendidas como cursos de acción y flujos de información relacio- 
nados con un objetivo público definido en forma democrática, que son desarrollados por el sector público, frecuentemente con la participación de la comunidad y el sector privado. «Una política pública de calidad incluirá orientaciones o contenidos, instrumentos o mecanismos, definiciones o modificaciones institucionales, y la previsión de sus resultados» (Lahera Parada, 2002).

Para cerrar aún más el universo de este trabajo y hacerlo más aprehensible, es necesario buscar una referencia teórica aún más precisa, una que defina qué es una política cultural. Teixeira Coelho (1997), en su Diccionario crítico de política cultural, la define como:

[...] programa de intervenciones realizadas por el Estado, instituciones civiles, entidades privadas o grupos comunitarios con el objetivo de satisfacer las necesidades culturales de la población y promover el desarrollo de sus representaciones simbólicas. Sobre este entendimiento inmediato, la política cultural se representa así como un conjunto e iniciativas tomadas por esos agentes, buscando promover la producción, distribución y el uso de la cultura, la preservación y difusión del patrimonio histórico y el ordenamiento del aparato burocrático responsable de ellas. Esas intervenciones asumen la forma de: 1) normas jurídicas, en el caso del Estado, o procedimientos tipificados, en relación a los demás agentes, que rigen las relaciones entre los diversos sujetos y objetos culturales, y 2) intervenciones directas en el proceso cultural propiamente dicho (construcción de centros de cultura, apoyo a manifestaciones culturales específicas, etc.). (p. 292)

Tomando como base argumental lo expuesto en los párrafos anteriores, salta a las claras que es el Estado, a través de sus políticas en cultura, el que debe velar por que ella se ejerza con libertad, creatividad y disfrute. ${ }^{1}$ Por tanto, las políticas públicas en cultura serán el «conjunto de principios, prácticas y presupuestos que sirven de base para la intervención de los poderes públicos en la actividad cultural, radicada en

\footnotetext{
La Declaración Universal de los Derechos Humanos (1948) establece en su artículo 27:

«- Toda persona tiene derecho a tomar parte libremente en la vida cultural de la comunidad, a gozar de las artes y a participar en el progreso científico y en los beneficios que de él resulten.

»- Toda persona tiene derecho a la protección de los intereses morales y materiales que le correspondan por razón de las producciones científicas, literarias o artísticas de que sea autora».

A su vez, en el Pacto Internacional de Derechos Económicos, Sociales y Culturales (1966), su artículo 6 dispone:

«- Entre las medidas que habrá de adoptar cada uno de los Estados Partes en el presente Pacto para lograr la plena efectividad de este derecho deberá figurar la orientación y formación técnicoprofesional, la preparación de programas, normas y técnicas encaminadas a conseguir un desarrollo económico, social y cultural constante y la ocupación plena y productiva, en condiciones que garanticen las libertades políticas y económicas fundamentales de la persona humana».
} 
su jurisdicción territorial, con el objeto de satisfacer las necesidades sociales de la población, en cualquiera de los sectores culturales» (UNESCO, 1982).

Estos son los principales fundamentos teóricos que dan la base a la tarea que se pretende llevar adelante en este trabajo. Incluso partiendo de una definición muy general de cultura, es impensable el desarrollo cultural de un país por fuera del accionar estatal. Ese accionar se basa en las políticas públicas en cultura, que lleva adelante un conjunto específico de instituciones que forman parte del Estado, a las que además se les asigna un presupuesto para su correcto desempeño. Aquí veremos solo una porción de las políticas públicas en cultura, la que corresponde al vínculo entre Presupuesto Nacional y la asignación presupuestal a las instituciones públicas con especificidad en la materia.

Existen dos formas de ver los datos financieros del Presupuesto Nacional en Uruguay. Una es aquella que los ordena desde una perspectiva funcional. Allí el presupuesto es asignado según áreas programáticas (AP), funciones superiores que realiza el Estado que van más allá de los períodos concretos de cada gobierno. A su vez, cada AP está compuesta por programas presupuestales, que son el conjunto de actividades o políticas mediante las cuales el Estado procura alcanzar un objetivo en un período estipulado, para lo que puede contribuir una institución o varias (OPP, s/f.b).

Uruguay tiene 18 áreas programáticas. La relativa a la cultura es «Cultura y deporte», que

[...] comprende la administración y prestación de tres diferentes tipos de servicios:

1. servicios culturales, los que además se supervisan y regulan. Dentro de ellos se incluye la gestión o apoyo de instalaciones para bibliotecas, museos, galerías de arte, teatros, salones de exposición, monumentos, edificios y lugares históricos, así como actos culturales de conciertos, producciones teatrales y cinematográficas, exposiciones de arte, entre otros;

2. asuntos relacionados con Servicios Editoriales, de Radio y Televisión. No incluye la supervisión ni la regulación de estos servicios prestados por privados, y, por último;

3. servicios recreativos y deportivos, así como la supervisión y regulación de instalaciones y actividades deportivas. (OPP, s/f.a) 
Figura 1. Asignación presupuestal por área programática

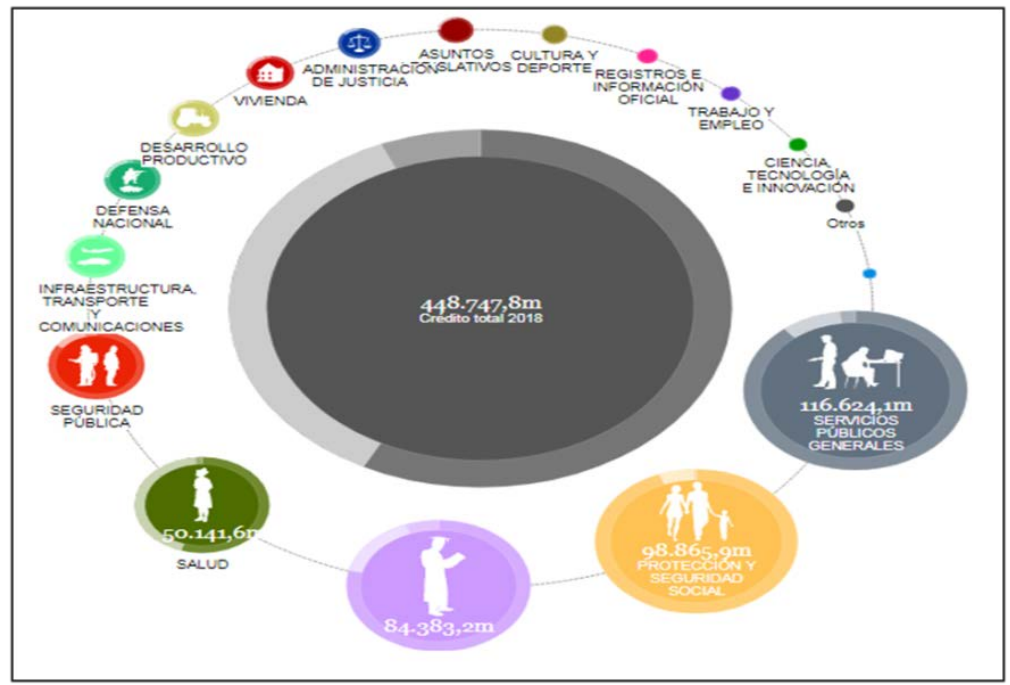

Fuente: Portal de Transparencia Presupuestaria, https://transparenciapresupuestaria.opp.gub.uy/inicio/presupuesto-nacional/a-d\%C3\%B3nde-vannuestros-impuestos.

A su vez, esta AP se descompone cuatro programas, de los que para este trabajo solo interesan dos:

1. Bienes y Servicios Culturales: Aumentar el nivel de producción y disfrute de los bienes y servicios culturales por parte de los ciudadanos, en todo el territorio nacional, protegiendo y promoviendo el desarrollo de los derechos culturales, la calidad y la diversidad cultural.

2. Institucionalidad Cultural: Contribuir al desarrollo, a la preservación y a la difusión del acervo cultural de las instituciones culturales públicas y privadas, así como el estímulo de la interacción público-privado. (oPP, s/f. a) ${ }^{2}$ 
Figura 2. Asignación presupuestal por programa

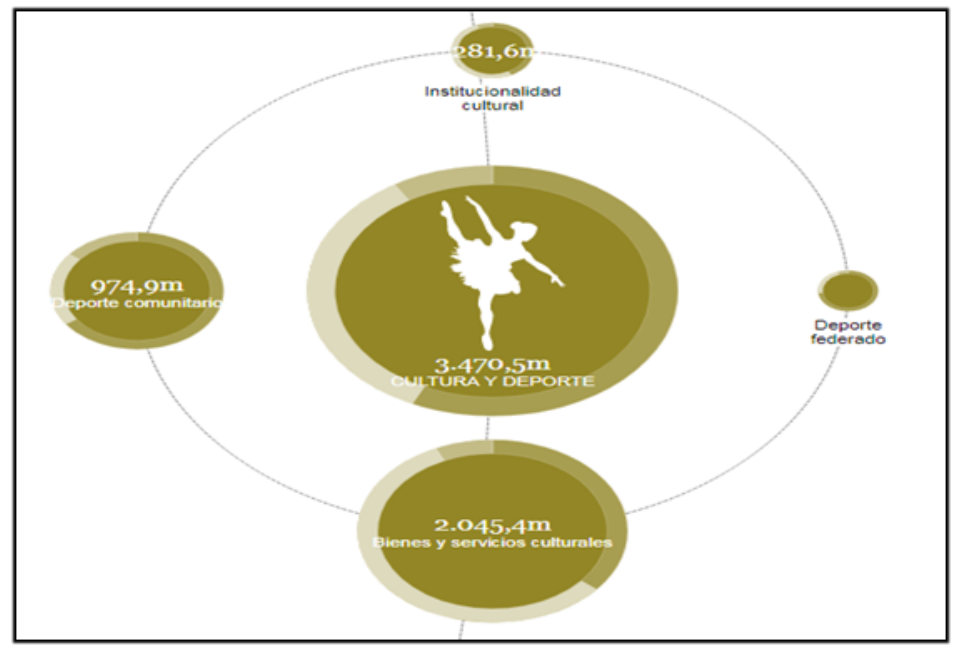

Fuente: OPP, s/f.b.

Esta primera perspectiva tiene la ventaja de dar información sobre áreas que trascienden la institucionalidad específica en tal o cual materia. El punto más importante que muestran los datos de esta área programática es que promedialmente, entre el 2011 y el 2018 — que es el período en el que funciona esta perspectiva de asignación presupuestal一, el 99,3\% del presupuesto asignado a estos dos programas que refieren a la cultura - Bienes y Servicios Culturales e Institucionalidad Cultural- se lo lleva el Ministerio de Educación y Cultura (MEC), ${ }^{3}$ en tanto el 0,07\% restante le es asignado al Ministerio de Transporte y Obras Públicas (MTOP) solo en el programa de Bienes y Servicios Culturales. Aquí se confirma algo que, por obvio en cuanto a misión y cometidos, pocas veces se justifica desde lo presupuestal: el MEC es el órgano rector en materia cultural. Sin embargo, el presupuesto para el accionar en esta materia es mucho más grande que el de estos dos programas, por lo que es necesario considerarlo desde la otra perspectiva.

La segunda forma de ver los datos financieros del Presupuesto Nacional es conocida como la perspectiva institucional.

Según ella el presupuesto se asigna, en un primer nivel, por categorías llamadas Incisos. La mayoría de los Incisos corresponde a organismos, como ministerios o agencias del gobierno nacional, así como al Poder Judicial y organismos de con- 
tralor electoral, financiero y administrativo. El segundo nivel de esta clasificación lo representan las Unidades Ejecutoras. Estas son subunidades organizativas cuyo jerarca tiene potestades de gestión de recursos financieros (ordenador del gasto). (OPP, s/f. b)

Las instituciones, correspondientes a unidades ejecutoras dentro del MEC, que se han elegido para este trabajo son:

- $\quad$ Dirección General de Secretaría (n. ${ }^{\circ}$ 1)

- Dirección Nacional de Cultura (DNC) (n. $\left.{ }^{\circ} 3\right)$

- Museo Histórico Nacional (n. ${ }^{\circ}$ 4)

- Museo Nacional de Historia Natural y Antropología (n. ${ }^{\circ} 6$ )

- $\quad$ Archivo General de la Nación (n. ${ }^{\circ}$ )

- Comisión del Patrimonio Cultural de la Nación (n. ${ }^{\circ}$ 8)

- Museo Nacional de Artes Visuales (n. ${ }^{\circ}$ 10)

- $\quad$ Dirección General de la Biblioteca Nacional (n. $\left.{ }^{\circ} 15\right)$

- $\quad$ Servicio Oficial de Difusión, Radiotelevisión y Espectáculos (Sodre) (n. 16)

- $\quad$ Servicio de Comunicación Audiovisual Nacional (n. $\left.{ }^{\circ} 24\right){ }^{4}$

Como todo recorte de la realidad, el que se presenta aquí es arbitrario y por fuera de él queda una infinidad de posibilidades. Así, solo a manera ilustrativa, han quedado fuera de este análisis aspectos como las bandas militares, los coros que funcionan en algunos ministerios, los bachilleratos artísticos o los distintos tipos de talleres que implican lo artístico-cultural y que existen entre las acciones que llevan adelante las diversas instituciones públicas de los organismos implicados en el Presupuesto Nacio-

Dos aclaraciones son fundamentales. La primera es que, dado el recorte temporal con el que se trabaja y los datos de los que se dispone, se incluye la Unidad Ejecutora Dirección General de Secretaría en su totalidad, a sabiendas de que es una decisión cuestionable. Sin embargo, no incluirlo sería tan cuestionable como hacerlo, dado que hasta 2008 su presupuesto incluía prácticamente todas las tareas y funcionarios de la Dirección de Cultura, así como de otras direcciones y unidades ejecutoras (muchos funcionarios son de esa UE aunque desempeñen tareas en otras, así como muchos de los que allí trabajan realizan tareas vinculadas al quehacer cultural). La decisión de incluir todo el presupuesto asignado a esta UE se hace con la intención de minimizar lo negativo que podría tener el no poder desagregar, por falta de datos, todas las actividades y los gastos de personal en cultura que ella ha tenido a lo largo de la serie que se propone estudiar.

La segunda aclaración es que no está incluida como unidad ejecutora la Dirección de Centros MEC (su número es el 05), creada por el artículo 120 de la Ley de Rendición de Cuentas y Balance de Ejecución Presupuestal correspondiente al ejercicio 2016 (ley 19.535), aprobada y publicada en setiembre de 2017. Los datos presupuestales de esta aún no se encuentran disponibles, ya que los recursos humanos, financieros y materiales y los créditos presupuestales asignados a la Dirección de Centros MEC en la unidad ejecutora 001, Dirección General de Secretaría, aún no han finalizado su proceso de transferencia de una a otra. 
nal, entre muchos otros ejemplos posibles. La imposibilidad de llegar a esos niveles de desagregación en los datos - para lo cual habría que hacer casi una tarea de arqueología - también habilita una ventaja comparativa: la de intentar centrar el foco en aquellos espacios que se dedican específicamente a la labor de las políticas culturales en la órbita estatal del Poder Ejecutivo. ${ }^{5}$

Esta perspectiva permitirá ver con mayor fidelidad la relación de la cultura con el Presupuesto Nacional, en tanto habilita a mirar cómo ha sido su asignación y ejecución por cada UE, más allá del área programática y los programas en particular. Esto es así porque muchas de esas UE llevan adelante políticas públicas en cultura y acciones que les implican más de un área programática, ya que, por ejemplo, muchas de las acciones que esas UE desarrollan implican el cruce de presupuestos de dos y tres áreas. ${ }^{6}$

Como fue dicho en la introducción, si bien existen datos de los tres tipos de gasto -funcionamiento, inversión y personal- hasta 1961, la información solo llega al nivel de desagregación de cada inciso (el MEC en este caso). En cambio, hasta 1999 sí existen datos de estos tres tipos de gasto desagregados para cada unidad ejecutora. Como lo que se propone este trabajo es vincular el presupuesto a las políticas públicas en cultura tal cual fueron definidas, esta información es la que se utilizará, en tanto dice cuánto fue asignado y ejecutado presupuestalmente por cada unidad ejecutora vinculada al desarrollo de esas políticas.

Sumado a esto, un corte que permita ver la evolución y los cambios presupuestales en los últimos 20 años ya salta a los ojos como un ejercicio más que interesante, especialmente cuando se comprueba que el punto de partida es el año de inicio de una de las crisis económicas más fuertes que sacudieron al país, abarca el último período de gobierno del Partido Colorado y los tres del Frente Amplio. Aunque no será el foco

5 Otras aclaraciones también son fundamentales para entender el encuadre de este trabajo. En primer lugar, quedan por fuera de este análisis todos aquellos gastos, inversiones, apoyos y/o esponsorización que realiza cualquiera de los entes autónomos y servicios descentralizados, por la misma razón: la imposibilidad de llegar a ciertos niveles de desagregación de la información que hace casi imposible la tarea para una persona sola. Aunque la información existe y está disponible, se requiere de un equipo que pueda recolectar datos de la forma más minuciosa posible, cruzando gastos e inversiones que a priori no son estrictamente culturales dado que esas instituciones no lo fundamentan de esa forma en términos presupuestales.

En este sentido ¿quién podría decir que el Antel Arena no es una infraestructura cultural y por tanto esa construcción es una inversión en cultura? Sin embargo, los montos de dinero destinados a ese fin posiblemente nunca serán contados como tal. Hay cientos de ejemplos similares, como la organización de festivales, la esponsorización o el apoyo a eventos de distinto tipo; pero también hay un sinfín de actividades minúsculas y de montos que pasarían prácticamente inadvertidos por cualquiera que se proponga semejante tarea, como puede ser la bajada de luz o el alquiler de generadores por UTE para las fiestas tradicionales, festejos de las localidades o celebraciones barriales.

6 Algunas de estas unidades ejecutoras tienen presupuestos de las áreas programáticas: Educación, Servicios públicos generales, Ciencia, tecnología e innovación y Desarrollo productivo. 
de trabajo aquí presentado, vale la pena tenerlo presente como contexto político general en el que se dieron los cambios que se mostrarán en las próximas páginas.

El foco del trabajo es un análisis descriptivo del lugar que ocupa la cultura en el Presupuesto Nacional a través de un repaso en la evolución de la asignación y la ejecución presupuestal en cultura según la perspectiva institucional, tomando las UE mencionadas más arriba para delimitar el campo de estudio.

\section{De la asignación a Cultura}

La primera aproximación a la asignación total que tiene la cultura —según hemos definido- dentro del Presupuesto Nacional consiste en observar su evolución a lo largo de los 20 años que se propone este trabajo. Para ello se ha pasado la asignación a pesos constantes con base en el 2010.

Gráfico 1. Presupuesto en cultura (en pesos constantes con base 2010)

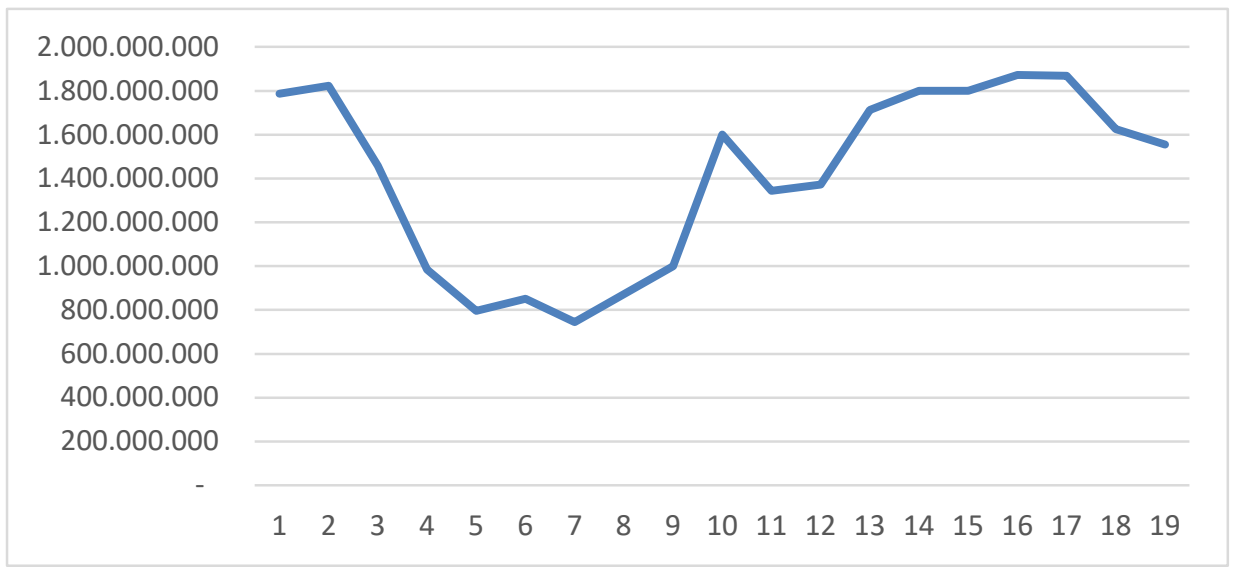

Fuente: Elaboración propia con datos de la Dirección de Gestión y Evaluación (AGEV) de la Oficina de Planeamiento y Presupuesto (OPP) de Presidencia de la República y el Instituto Nacional de Estadística (INE).

En el gráfico 1 se puede ver claramente una baja muy abrupta con la crisis económica 1999-2002 que afectó a la sociedad uruguaya, con la que era dable esperar una merma significativa en la asignación presupuestal en cultura —al igual que en otros muchos sectores- A partir del 2005 se advierte una recuperación, con un pico fuerte que llega en el 2008 y es acompañado de una baja en el 2009 y una recuperación gran- 
de a partir del 2011 hasta el 2014, que es el punto más alto de la serie; desde allí comienza un nuevo período de baja que sigue hasta el 2017.

Los años de mayor asignación presupuestal para la cultura en la serie aquí presentada fueron el 2000 y el 2014, este apenas 2,62\% mayor que aquel. Quizá el apunte más importante de esta información es que a Uruguay le llevó 14 años alcanzar una asignación presupuestal en cultura similar a la que había cuando se iniciaba la crisis económica mencionada (cuyos impactos más sobresalientes y notorios fueron en el 2002), pero que pasado un año no pudo sostenerla y la asignación volvió a caer durante tres años consecutivos.

Aunque el gráfico anterior provee de información crucial a los efectos de este trabajo, una lectura clave y sumamente interesante es la que vincula la asignación presupuestal a cultura con el producto interno bruto (PIB). Esta da la pauta de si la primera ha acompañado los momentos de declive y auge económico por los que ha pasado el país desde 1999 hasta el 2017.

Gráfico 2. Asignación presupuestal a cultura como porcentaje del PIB (en pesos corrientes de cada año)

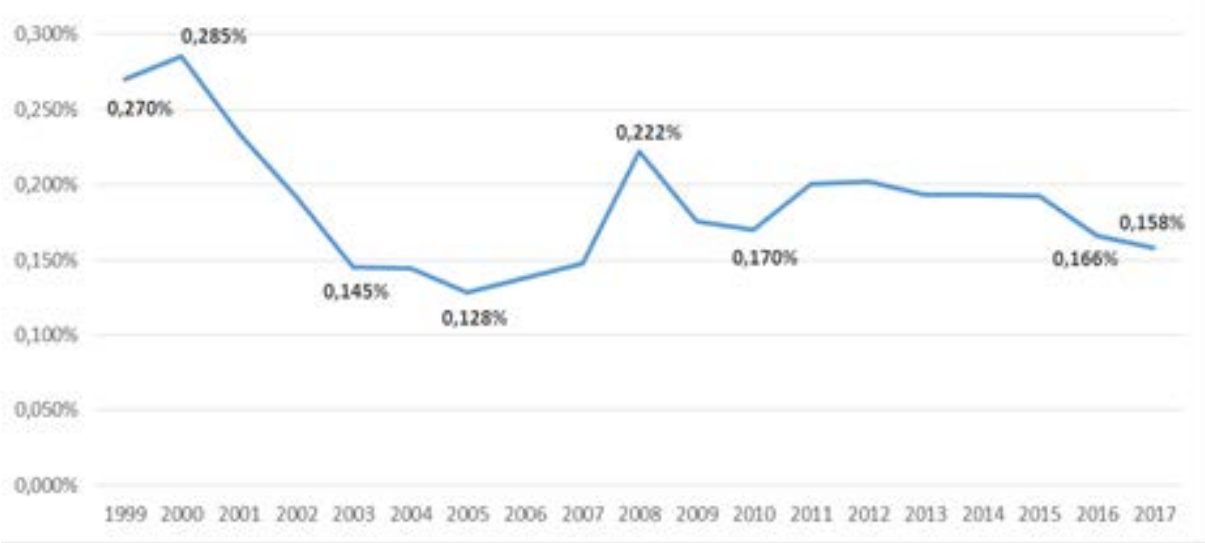

Fuente: Elaboración propia con datos de AGEV y el Banco Central del Uruguay (BCU).

Posiblemente lo primero que salte a la vista es el muy bajo porcentaje de asignación presupuestal para la cultura en relación con el PIB a lo largo de toda la serie histórica. El año 2000 fue el mejor, aunque la asignación ni siquiera llegó a representar el 0,3\% del PIB de ese año. Si a esto se suma que aproximadamente desde el 2002 la UNES- 
Co recomienda que el presupuesto público en cultura sea del 1\% del PIB, estos valores se hacen aún más bajos.

Saliéndonos en parte de esta cuestión, que desde siempre ha sido un reclamo del sector cultural, llaman la atención tres momentos en el período en los que la asignación presupuestal fue bastante mayor que en el resto: los años 1999 y 2000 son los de mayor asignación con relación al PIB, luego viene un declive hasta el 2005, cuando encuentra su punto más bajo $(0,128 \%$ del PIB), un repunte que alcanza su pico más alto en el $2008(0,222 \%)$ y una baja que se transforma en una especie de meseta entre el 2009 y el 2015, con valores entre 0,170\% (año 2010) y 0,202\% (año 2012); a partir de allí se hay una baja que llega a $0,158 \%$ en el 2017.7

Era esperable que la crisis económica 1999-2002 afectara la asignación presupuestal en cultura; sin embargo, llama más la atención que entre el 2003 y el 2017, con 15 años de crecimiento del PIB, que en promedio fue del 4,34\%, la asignación a la cultura haya tenido esas oscilaciones y además nunca haya recuperado los valores previos a la crisis.

Yendo un poco más allá, la mirada complementaria es la del lugar que ocupa la asignación presupuestal a las UE especializadas en cultura en el Presupuesto Nacional. La intención con ella es mirar el lugar que ocupa la cultura dentro del marco general de asignación presupuestal.

Gráfico 3. Asignación presupuestal a cultura en el Presupuesto Nacional

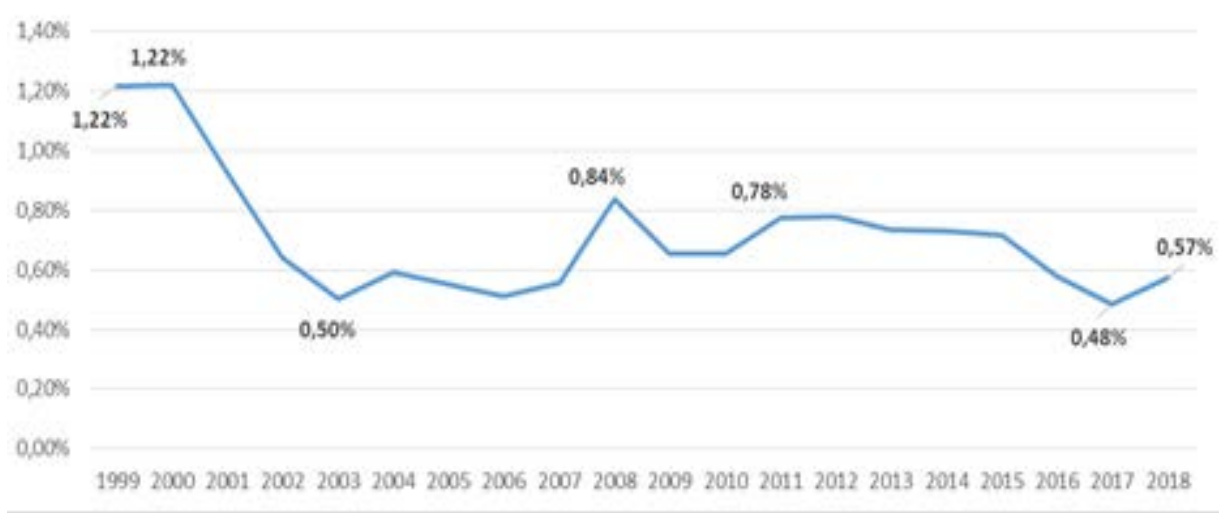

Fuente: Elaboración propia con datos de AGEV.

7 Cabe apuntar que los datos sobre PIB disponibles para los años 2016 y 2017 por el Banco Central del Uruguay aún son preliminares al escribir este artículo. 
Posiblemente salte a la vista que la forma de este segundo gráfico es muy similar a la del porcentaje del PIB que representa la asignación presupuestal a cultura. Desde el 2001 hasta la fecha, la asignación presupuestal a cultura no ha llegado a constituir el $1 \%$ del total de asignación del Presupuesto Nacional. Además, se observan los mismos momentos de auge presupuestal $(1999,2000$ y 2008), así como se comprueban casi los mismos momentos de declive, con la gran salvedad del 2017, que presenta números por debajo del peor momento histórico de la serie (2003), en pleno proceso de recuperación de la crisis económica mencionada.

Si bien esto no debe generar una alarma sin asidero en la realidad, puesto que la Ley de Rendición de Cuentas del 2017 aún no está aprobada, sí debe alertarse acerca de un proceso de baja pronunciada en el presupuesto asignado a la cultura desde el 2015 al 2017. Aunque los números de este último año sean algo mejores que lo aquí expresado, las diferencias no serán lo suficientemente significativas para contradecir el proceso de baja; incluso el aumento que se percibe en el 2018 está por debajo de la asignación que tuvo la cultura en el 2016 (0,58\%).

\section{La asignación presupuestal en cultura: una mirada al reparto interno}

Tomando como punto de partida la afirmación de que el MEC es el órgano rector en materia de política pública en cultura, con la fundamentación presupuestal que sumamos en términos de áreas programáticas, una mirada al reparto presupuestal dentro de dicho ministerio permite comprobar que no solo es el órgano rector, sino que en términos presupuestales es un Ministerio de Cultura y muchas otras cosas. Aunque no en términos de presupuestos, sino de tareas, políticas y acciones, algo de esto decía Gonzalo Carámbula en su texto «La institucionalidad cultural pública como problema» (2011).

En 16 de los 20 años que se trabajan en este documento, salvo en el período de recuperación económica poscrisis (2003-2007), la asignación a cultura en el presupuesto del MEC ha sido siempre superior al 50\% del total, con un promedio del $61 \%$ entre el 2008 y el 2018, cuando ha encontrado una especie de meseta con oscilaciones poco pronunciadas.

Sin embargo, y como es lógico, no todas las UE tienen la misma participación en esas asignaciones totales de montos presupuestales. El gráfico 5 presenta los porcentajes de asignación que ha tenido cada UE en los últimos 20 años sobre el presupuesto total asignado a cultura. 
Gráfico 3. Asignación presupuestal a cultura en el presupuesto asignado al MEC

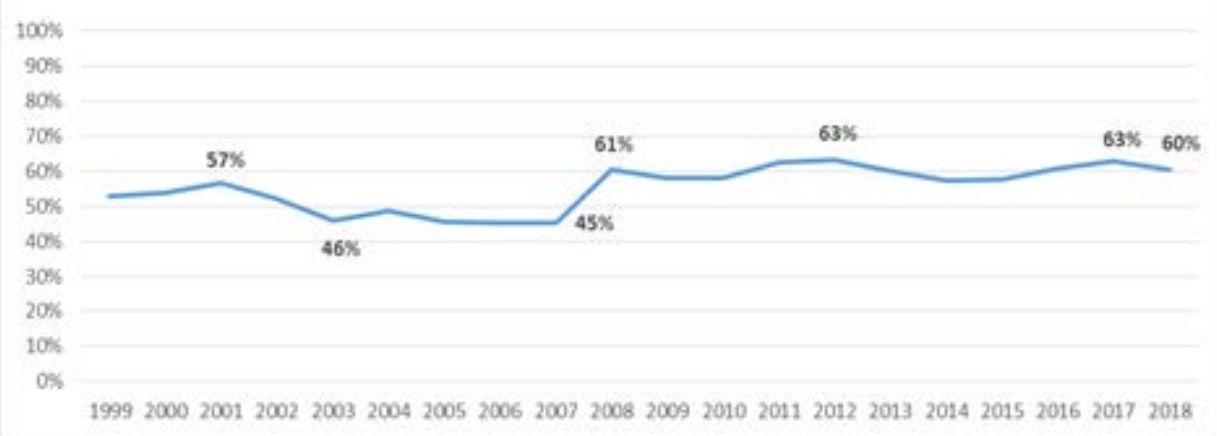

Fuente: Elaboración propia con datos del Portal de Transparencia Presupuestaria de la opP de Presidencia de la República.

Gráfico 5. Asignación presupuestal a cada unidad ejecutora respecto al total de la asignación a cultura

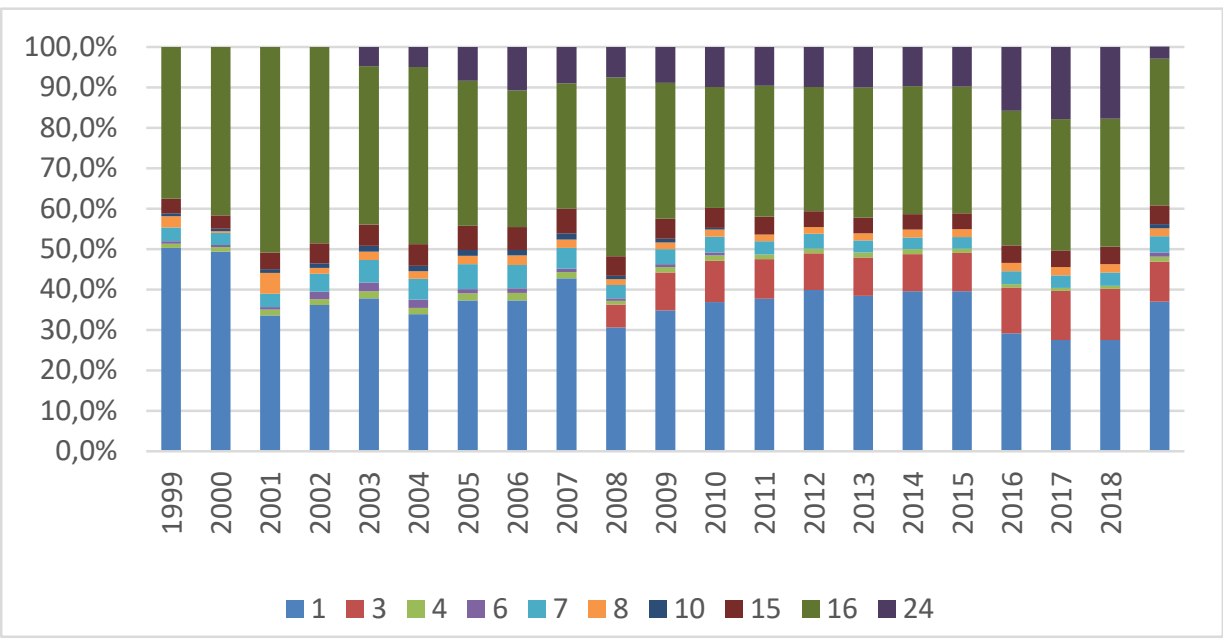

Nota: Cada número corresponde a una unidad ejecutora: 1) Dirección General de Secretaría; 3) Dirección Nacional de Cultura; 4) Museo Histórico Nacional; 6) Museo Nacional de Historia Natural y Antropología; 7) Archivo General de la Nación; 8) Comisión del Patrimonio Cultural de la Nación; 10) Museo Nacional de Artes Visuales; 15) Dirección General de la Biblioteca Nacional; 16) Sodre; 24) Servicio de Comunicación Audiovisual Nacional.

Fuente: Elaboración propia con datos del Portal de Transparencia Presupuestaria de la opP de Presidencia de la República. 
La Dirección General de Secretaría (UE 1) es históricamente la que mayor asignación presupuestal ha tenido, con un promedio de 37,1\% en los últimos 20 años; sin embargo, como fue dicho más arriba, los datos sobre ella no discriminan lo que es específicamente cultural (es decir, contempla otras tareas más allá de las culturales, ya que no es posible desagregarlas). Muy de cerca lo secunda el Sodre (UE 16), con un promedio de $36,3 \%$ en las últimas dos décadas; esto es sumamente significativo por su carácter multifacético en el ejercicio de las políticas culturales. Tanto que el servicio de televisión pública estaba inserto en las tareas que desarrollaba el Sodre, hasta que en el 2003 se creó la UE 24, primero para el Canal 5 TVEO, que luego devino en el Servicio de Comunicación Audiovisual Nacional (SECAN), de acuerdo con la ley 19.307, de diciembre del 2014, que lo creó. ${ }^{8}$

Como dice María Inés de Torres (2015), históricamente el diseño del Sodre ha sido muy peculiar. Ella lo llama «de doble vía», por cuanto supuso la instalación al mismo tiempo de la radio y del Estudio Auditorio, ${ }^{9}$ por lo que podría decirse que recién a partir de la creación del SECAN el Sodre se ha concentrado en su Estudio Auditorio (cuerpos estables, ${ }^{10}$ Archivo de la Imagen y la Palabra, escuelas de formación artística y los auditorios).

El mayor porcentaje de asignación presupuestal para la UE del Sodre se registró en el 2001, con el 50,8\%, pero, como fue dicho, en ese momento el servicio de televisión pública estaba inserto en él, por lo que ese porcentaje no admite una comparación temporal. En 2008, con la UE 24 ya creada, el Sodre tuvo una asignación del 44,2\% sobre el total, pero el servicio de Radiodifusión Nacional de Uruguay aún funcionaba dentro de él. Por tanto, los mejores datos para comparar son los del 2015 en adelante, ya creado el SECAN. Desde entonces, el año de mayor asignación presupuestal para el Sodre fue el 2017, con el 32,5\%, y también fue el de mayor asignación para la UE 24 (SECAN), con el 17,8\%. Ese año también fue el de mayor asignación para la DNC (UE 3) desde su creación, en el 2007, y su primera asignación presupuestal, en el 2008, con un $12,6 \%$.

Las cuatro UE mencionadas - Dirección General de Secretaría (1), Dirección Nacional de Cultura (3), Sodre (16) y SECAN (24) - explican el 89,5\% del presupuesto asignado a cultura en el 2018; de ahí su destaque en el párrafo anterior. Sin embargo, tanto el Museo Nacional de Artes Visuales (UE 4), con un promedio de asignación presupuestal de 1,2\%, el Archivo General de la Nación (UE 7), con un promedio de 3,9\%, la Nacional de Uruguay (RNU, antes Radios Públicas del SODRE).

9 Recordemos que fue creado en diciembre de 1929 por la ley 8.557. En abril de 1930 hizo la primera emisión radial y solo un año después se creó la Orquesta Sinfónica, el primer cuerpo estable.

10 Orquesta Sinfónica, Ballet Nacional, Coro Nacional, Conjunto de Música de Cámara y Coro Nacional de Niños. 
Comisión del Patrimonio Cultural de la Nación (UE 8), con un promedio de $2 \%$, y la Dirección General de la Biblioteca Nacional (UE 15), con un promedio de 4,6\%, han tenido una permanencia de largo aliento en la institucionalidad cultural y presupuestal del país y todo parece indicar que así seguirá. La excepción entre ellos es el Museo Histórico Nacional (UE 4), que paulatinamente ha pasado a funcionar en la órbita de la DNC, en un proceso iniciado en diciembre del 2012 a partir de la ley 19.037, que creó el Sistema Nacional de Museos, y es coordinado por dicha dirección según establece el decreto reglamentario 295/14 de la Presidencia de la República. ${ }^{11}$

Si tomamos la definición de institucionalidad cultural como el «conjunto de los organismos públicos y privados, como los instrumentos legales, financieros, de infraestructura y de gestión que tanto el Estado como la sociedad civil crean y ponen al servicio del desarrollo artístico y cultural y de la conservación y difusión del patrimonio cultural del país, tanto en los niveles internacional, regional, nacional, comunal y barrial en que se desenvuelve la vida de las personas [...] [aunque] en sentido estricto, se utiliza para aludir únicamente a los organismos públicos y privados que cumplen funciones y procuran conseguir objetivos en el campo cultural» (Carámbula, 2011), y nos concentramos en los organismos públicos, a priori pensaríamos en cierta inamovilidad de las estructuras. Sin embargo, algo que se vislumbra en los datos presentados es que esa inamovilidad es solo aparente y que en los últimos 20 años las instituciones públicas con especificidad en lo cultural se han ido modificando e intentando adaptarse a los tiempos actuales. Esta idea se refuerza si se observa que el MEC ha procurado desconcentrar sus labores tramitando mayores asignaciones presupuestales a sus UE que están por fuera de la órbita central, es decir, por fuera de la Dirección General de Secretaría.

Ahora bien, con una mirada al tipo de gasto que han tenido estas UE es posible hacerse una idea general de en qué se ocupa ese porcentaje presupuestal del MEC tan alto que lo hace eminentemente cultural.

Como se mencionó, el Presupuesto Nacional distingue tres tipos de gasto: en personal, en funcionamiento y en inversión. Los primeros son las remuneraciones, incluidos los aguinaldos, cargas legales y prestaciones sociales. El gasto en funcionamiento comprende todas las compras y arrendamientos de bienes y servicios no personales que sirven para el funcionamiento cotidiano de los organismos; también las erogaciones por suministros prestados por las empresas públicas o paraestatales (agua, líneas telefónicas, electricidad, combustible, etcétera), así como las subvenciones, subsidios y otras transferencias a otros sectores. Por gasto en inversión se entiende toda aplicación de recursos a cualquier tipo de bien o actividad que incremente el patrimonio 
físico de los organismos comprendidos en el Presupuesto Nacional, los estudios de inversión y excepcionalmente también la capacitación de los funcionarios. ${ }^{12}$

Gráfico 6. Presupuesto en cultura según el tipo de gasto

(en pesos constantes con base 2010)

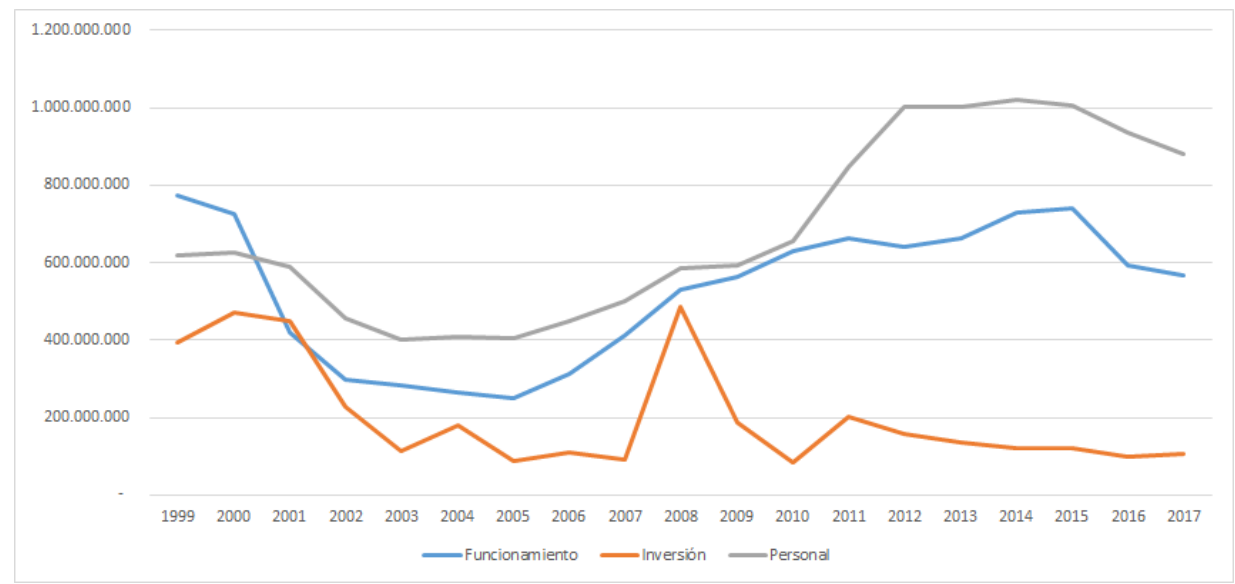

Fuente: Elaboración propia con datos del Portal de Transparencia Presupuestaria de la OPP y del INE.

Los datos expresados en este gráfico dan cuenta de tres fenómenos interesantes. El primero es que el presupuesto de las UE especializadas en el quehacer cultural asignado para hacer inversiones tuvo su pico en el 2008, luego una baja significativa, una pequeña recuperación en el 2011 y de allí en adelante una baja continuada, aunque menos pronunciada que las que anteriores. Este fenómeno es el que explica el pico presupuestal en cultura como porcentaje tanto del PIB $(0,222 \%)$ como del Presupuesto Nacional $(0,84 \%)$ para el 2008 , así como parte de la meseta y la tendencia a la baja que se registra a partir del 2011 en ambos casos.

El segundo fenómeno es que el gasto en funcionamiento aumentó progresivamente entre el 2005 y el 2015 (con excepción del 2012) y a partir de allí ha tenido una merma bastante considerable, lo que contribuye también a explicar una parte de la baja presupuestal general presentada como porcentaje del PIB y como porcentaje del Presupuesto Nacional.

12 Para ampliar esta información, se sugiere consultar la «Nota Metodológica - PN» disponible en el Portal de Transparencia Presupuestaria de la Oficina de Planeamiento y Presupuesto (www.transparenciapresupuestaria.opp.gub.uy). 
El tercer fenómeno es que los gastos en personal aumentaron progresivamente, también desde el 2005, con un salto incremental a partir del 2010 y una baja a partir del 2015, todo lo cual redunda también en la meseta con tendencia a la baja en la asignación presupuestal a cultura como porcentaje del PIB y como porcentaje del Presupuesto Nacional.

Por su parte, la baja en los gastos de funcionamiento a partir del $2015 \mathrm{y}$ el salto incremental y baja del gasto en personal a partir del 2011 y el 2015 respectivamente son fenómenos íntimamente relacionados. El primero se explica a partir de una situación que comienza con el segundo. Es decir, con la Ley de Presupuesto Nacional 18.719, aprobada en diciembre del 2010 (artículos 498 y 501) y los decretos presidenciales 52, 53 y 55, de febrero del 2011, que corrigen las formas de «contrato artístico», crean el «contrato laboral» y el «contrato temporal de derecho público», ${ }^{13}$ comienza un proceso de regularización contractual de diversas situaciones que tenían muchos años en las UE especializadas en cultura. Este proceso de regularización alcanzó su punto más alto en el 2015, cuando se produjo un ingreso definitivo a la presupuestación, mediante pruebas y/o concursos (internos o abiertos), de la mayor parte del personal que venía arrastrando situaciones contractuales irregulares, defectuosas o contradictorias con los mencionados decretos; esto, entre otras cosas, implicó que se liberara y bajara el gasto en funcionamiento, al tiempo que se mantuvieran iguales los montos corrientes del gasto en personal (por ello al ponerlo en pesos constantes se puede ver una baja menos pronunciada que para el gasto en funcionamiento).

Si miramos la asignación presupuestal en funcionamiento por unidad ejecutora vemos los siguientes datos según el gráfico 7.

13 Para ver estos decretos en detalle se sugiere ingresar al siguiente link: https://www.onsc.gub.uy/ onsc1/index.php?option=com_content\&view=article\&id=150:se-aprueban-decretos-reglamentariossobre-vinculos-laborales-y-el-sistema-de-reclutamiento-y-seleccion\&catid=1:latest-news (30 de marzo de 2018). 
Gráfico 7. Asignación a funcionamiento por UE respecto al total de asignación a funcionamiento en Cultura

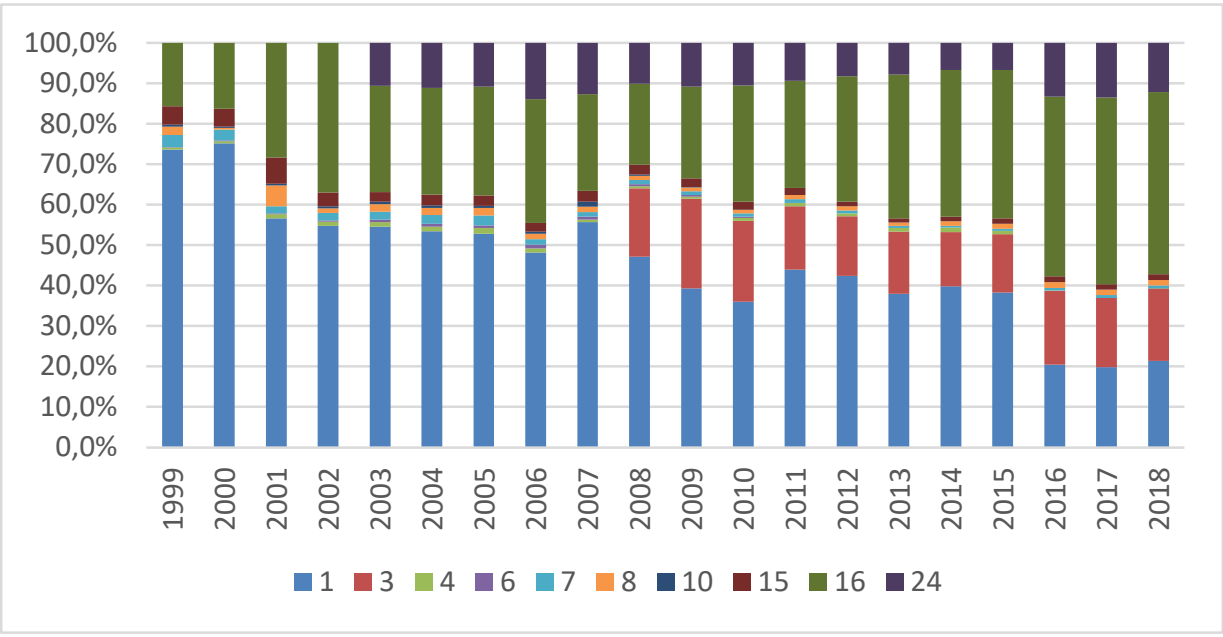

Nota: Cada número corresponde a una unidad ejecutora: 1) Dirección General de Secretaría;

3) Dirección Nacional de Cultura; 4) Museo Histórico Nacional; 6) Museo Nacional de Historia Natural y Antropología; 7) Archivo General de la Nación; 8) Comisión del Patrimonio Cultural de la Nación;

10) Museo Nacional de Artes Visuales; 15) Dirección General de la Biblioteca Nacional; 16) Sodre;

24) Servicio de Comunicación Audiovisual Nacional.

Fuente: Elaboración propia con datos del Portal de Transparencia Presupuestaria de la oPP de Presidencia de la República.

Con estos datos se puede ver que las UE que explican el mayor porcentaje de asignación presupuestal son en general también las que tienen los mayores porcentajes de asignación en funcionamiento. Las cuatro contratan diversos tipos de profesionales en las artes y la cultura, otorgan premios, financian eventos y organizan los suyos; todos estos son gastos de ese tipo (entre otros).

Aquí también se puede apreciar el fenómeno de desconcentración presupuestal que viene llevando adelante el MEc desde la Dirección General de Secretaría hacia otras UE. Así por ejemplo, en 1999 la asignación presupuestal a la UE 1 era del 73,3\% del total, y en el 2018 es del 21,3\%; se puede ver además que este fenómeno es más profundo a partir de la Ley de Presupuesto Nacional 19.355, aprobada en diciembre del 2015. Esto es fundamental por cuanto el gasto en funcionamiento es el principal para la gestión de las políticas, ya que es uno de los dos tipos de gasto más flexibles (el otro es inversión); o sea, es de los que con mayor facilidad se pueden asignar a ciertas acciones durante un período, en el marco de esas políticas, y luego asignarlo a otro tipo de acciones durante otro período sin que ello implique grandes complicaciones (Be- 
rretta, 2004). ${ }^{14}$ Es el más significativo tanto en términos relativos como en términos absolutos.

Otro fenómeno que también se advierte en este gráfico y que tiene continuidad con lo mencionado en los apartados anteriores es que el Sodre es la UE que tiene el mayor porcentaje de asignación. Esa mayoría sobre todo se debe a un aumento progresivo en la asignación entre el 2008, cuando tenía un 19,9\%, y el 2018, cuando tuvo un $45 \%$ del total.

Un punto que llama la atención es la falta de asignación presupuestal adicional para la Biblioteca Nacional en el 2016, bicentenario de su fundación. La asignación fue del 1,5\% del total, por debajo de su promedio de los últimos 20 años, que ha sido de $2,4 \%$.

Gráfico 8. Asignación a inversión por UE respecto al total de asignación a inversión en cultura

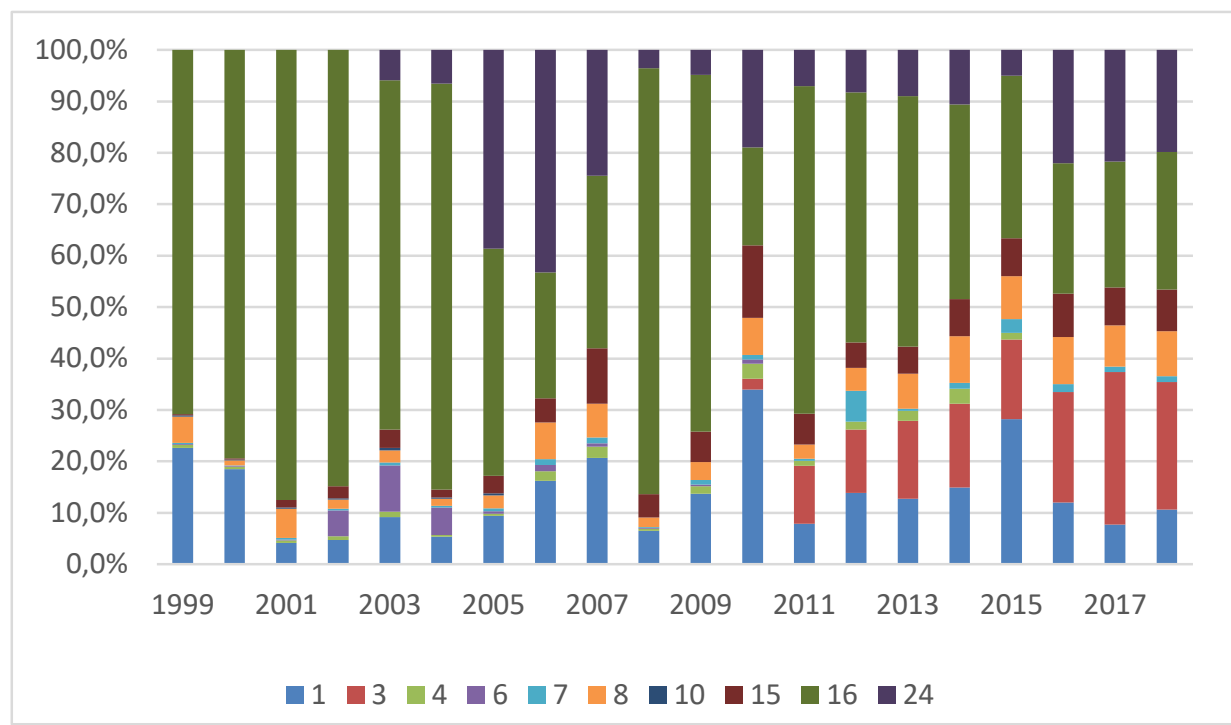

Nota: Cada número corresponde a una unidad ejecutora: 1) Dirección General de Secretaría;

3) Dirección Nacional de Cultura; 4) Museo Histórico Nacional; 6) Museo Nacional de Historia Natural y Antropología; 7) Archivo General de la Nación; 8) Comisión del Patrimonio Cultural de la Nación;

10) Museo Nacional de Artes Visuales; 15) Dirección General de la Biblioteca Nacional; 16) Sodre;

24) Servicio de Comunicación Audiovisual Nacional.

Fuente: Elaboración propia con datos del Portal de Transparencia Presupuestaria de la opp de Presidencia de la República.

14 Sin lugar a dudas, el tipo de gasto presupuestal más rígido es el de personal, dado que se trata de salarios en la órbita pública, donde los funcionarios presupuestados tienen entre sus derechos la inamovilidad del cargo. 
Viendo el gasto en términos de inversión, se comprenden algunos puntos que llamaron la atención en páginas anteriores, al tiempo que aparecen algunos fenómenos nuevos.

Lo primero - un fenómeno ya recurrente- es que la UE del Sodre es la que históricamente ha tenido la mayor asignación presupuestal en términos de inversión, con un promedio de 52,5\% del total en los últimos 20 años. Entre 1999 y el 2001, los números explican el primero de los picos de la asignación presupuestal total, presentados como porcentaje del PIB y como porcentaje del total del Presupuesto Nacional, por las obras de la Sala Hugo Balzo y la culminación del llamado cerramiento acústico de la sala principal (Eduardo Fabini) del Auditorio Adela Reta. El pico del 2008, que también se ha visto en gráficos anteriores, se explica en buena medida por la asignación para la culminación de la obra del mencionado auditorio. El fenómeno de la desconcentración se mantiene, aunque esta vez el movimiento es desde el Sodre al resto de las UE.

A su vez, las tareas de la Comisión del Patrimonio Cultural de la Nación (UE 8) explican cierto destaque en este tipo de gasto, con un pico de 9,2\% en el 2016 y un promedio de $5,2 \%$ en el total de la serie.

Destacables son los altos porcentajes de asignación para la UE 24 entre el 2005 y el 2007 (38,6\%, 43,3\% y 24,4\% respectivamente), que se explican por un proceso de actualización TNU que incluyó obras en los estudios de televisión y compra de equipamiento. Algo similar, aunque en menor medida, se podría verificar a partir del 2015 con la creación del SECAN. El promedio de la UE 24 desde su creación en el 2002 es del $15,6 \%$ del total del presupuesto asignado para inversiones, lo que la ubica en el segundo lugar detrás del Sodre.

La Biblioteca Nacional de Uruguay (UE 15) tuvo su inyección más fuerte de dinero para inversión en el 2010, con un 14,1\% del total, ${ }^{15}$ al comienzo del segundo período de gobierno del Frente Amplio. Se procuraba cubrir un largo período de desatención edilicia, por lo que la asignación fue sobre todo para reparaciones (techos, claraboyas, sistema de ventilación, etcétera), reforma (construcción de rampa para la accesibilidad universal) y actualización informática, entre otras. ${ }^{16}$

El último punto es el de la DNC, que a partir del 2011 ha tenido un paulatino crecimiento en su asignación presupuestal en este tipo de gasto. Buena parte de este crecimiento se explica por la trasposición, en el 2011, de la asignación presupuestal del Fondo para el Desarrollo de Infraestructuras Culturales en el Interior del país desde la

15 Su promedio en los últimos 20 años es del 5,4\% del total en este tipo de gasto.

16 Algo de la información de este proceso está disponible en: http://www.bibna.gub.uy/innovaportal/v/ 35628/4/mecweb/la_biblioteca_nacional?parentid=29757 (marzo, 2018). 
Dirección General de Secretaría hacia la DNC, que es la institución que lo gestiona y ejecuta. ${ }^{17}$

El último tipo de gasto que destaca es el de personal, que básicamente muestra movimientos que se han venido verificando en las páginas anteriores.

Gráfico 9. Asignación a personal por UE respecto al total de asignación a personal en Cultura

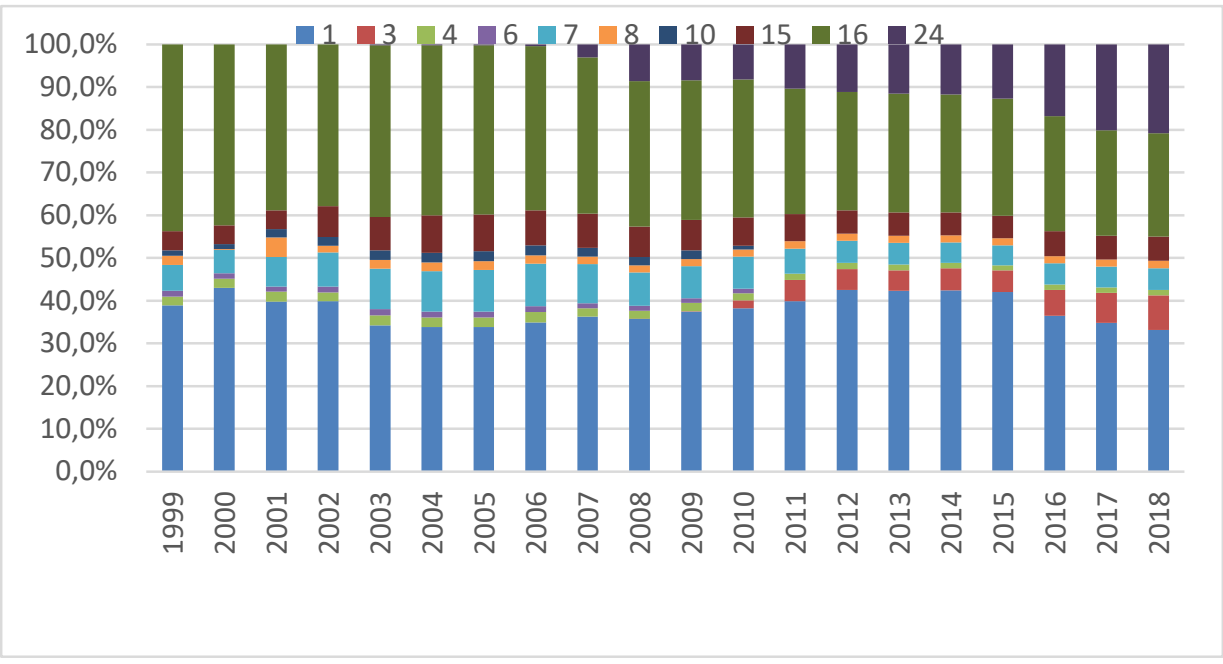

Nota: Cada número corresponde a una unidad ejecutora: 1) Dirección General de Secretaría; 3) Dirección Nacional de Cultura; 4) Museo Histórico Nacional; 6) Museo Nacional de Historia Natural y Antropología; 7) Archivo General de la Nación; 8) Comisión del Patrimonio Cultural de la Nación;

10) Museo Nacional de Artes Visuales; 15) Dirección General de la Biblioteca Nacional; 16) Sodre;

24) Servicio de Comunicación Audiovisual Nacional.

Fuente: Elaboración propia con datos del Portal de Transparencia Presupuestaria de la opP de Presidencia de la República.

El Archivo General de la Nación, que hasta ahora prácticamente no aparecía, es una de las UE más intensivas en presupuesto de personal en función de las políticas que desarrolla, con un promedio de asignación del 6,9\% en los últimos 20 años.

Otro punto que llama la atención es el de la UE 24 del SECAN, que en el 2018 se llevó un $20,8 \%$ de la asignación presupuestal. Esto también se explica en que las institu-

17 La otra parte grande de este tipo de gasto en la DNC es inversión en «equipamiento y mobiliario de locales culturales». 
ciones que lo componen y el servicio que se brinda a la ciudadanía son sumamente intensivos en gastos de personal con las estructuras actuales.

Otro punto destacable es que la UE 1, Dirección General de Secretaría, tiene el mayor promedio de asignación presupuestal en personal a lo largo de toda la serie, con $38 \%$, secundada por la UE 16, Sodre, con un 33,6\%. En el caso de la primera, la razón es que buena parte de los trabajadores de la cultura son de esa UE (la 1) aunque desempeñen tareas en otro lugar; en el caso de la segunda, su estructura y dimensiones de antaño explican su cuantioso porcentaje promedio de gasto en personal, que en el 2018, en su momento más bajo, alcanza el 24,2\% del total. Recordemos que el Sodre conserva sus cuerpos estables, los técnicos asociados a ellos y al auditorio, y tiene en sus filas distintos tipos de gestores y profesionales de la cultura.

\section{De la ejecución presupuestal}

Un punto aparte debe considerarse para la ejecución. Partiendo de la base de que ella nos habla de la eficiencia de ese inciso o unidad ejecutora para llevar adelante sus políticas en términos de presupuesto, una mirada a lo que ha hecho el MEC y en particular las unidades ejecutoras de cultura que reciben más asignación presupuestal es lo que se propone en este breve apartado.

Gráfico 10. Ejecución presupuestal en relación con la asignación presupuestal

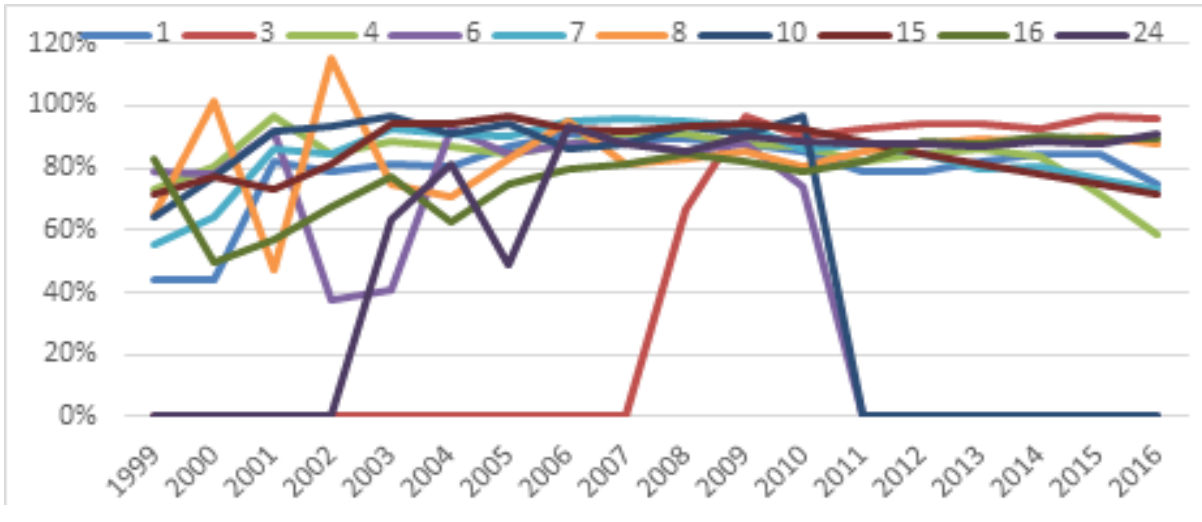

Nota: Cada número corresponde a una unidad ejecutora: 1) Dirección General de Secretaría;

3) Dirección Nacional de Cultura; 4) Museo Histórico Nacional; 6) Museo Nacional de Historia Natural y Antropología; 7) Archivo General de la Nación; 8) Comisión del Patrimonio Cultural de la Nación; 10) Museo Nacional de Artes Visuales; 15) Dirección General de la Biblioteca Nacional; 16) Sodre; 24) Servicio de Comunicación Audiovisual Nacional.

Fuente: Elaboración propia con datos del Portal de Transparencia Presupuestaria de la opP de Presidencia de la República. 
Todas las UE que se han trabajado en este artículo tienen promedios de ejecución del 77\% en adelante; de hecho, solo tres se encuentran por debajo del 80\%. La de más baja ejecución es el Museo Nacional de Historia Natural y Antropología (UE 6), que en el 2002 y el 2003 tuvo solo un 38\% y un 41\% de ejecución respectivamente, lo que le baja muchísimo la media. ${ }^{18}$

Los otros porcentajes de ejecuciones más bajos los tienen el Sodre (78\%) y la Dirección General de Secretaría (79\%). Esto se explica sobre todo por el tipo de gasto en personal, donde las vacantes dejan a la vista el espacio de no ejecución, ya que ese tipo de gasto es el más inflexible y menos sujeto a cambios.

El mejor porcentaje de ejecución lo tiene la DNC, con el 91\%, seguido por el Museo Nacional de Artes Visuales (UE 10), con el 89\%, pero recordemos que a partir del 2011 el segundo pasó a ser parte de la primera, por lo que no tiene más presupuesto propio.

Ahora bien, tomar estos datos de ejecución presupuestal como base para hablar de la eficiencia de las políticas culturales sería un error garrafal, pero no hacerlo es una omisión que quienes hacen gestión cultural no pueden permitirse.

La primera parte de la afirmación se funda en que, por la manera que se arma el Presupuesto Nacional uruguayo, la eficiencia en la ejecución está asociada a una serie de objetivos y metas que son estipulados o propuestos por los organismos que llevan adelante las políticas en cuestión. A su vez, esos objetivos y metas tienen una serie de indicadores, también propuestos por esos organismos, que deben ser mensurables cuantitativamente. La dificultad para medir la eficiencia de las políticas en estos términos es justamente la determinación de qué es lo mensurable. Establecer que las políticas culturales son más eficientes por tener cierta cantidad de actividades de circulación cultural, enviar un determinado número de artistas al exterior, hacer un número preestablecido de funciones para niños/as, agrandar o actualizar en tecnología un auditorio u otro es algo muy discutible.

Aunque es de Perogrullo y aparece como voz insistente en el sector cultural, y sin pretender que estas líneas se apeguen a la idea del buonismo de la cultura, que entiende «que todo aquello vivo o inerte que consigue adjudicarse el epíteto de cultural es bueno» (Rausell, Abeledo Sanchís, Carrasco Arroyo y Martínez Tormo, 2007, p. 21), sí es fundamental dejar en claro que hay una dimensión inconmensurable de lo cultural y en particular de la eficiencia o no de una política cultural. Esa dimensión trasciende por mucho cuánto presupuesto de ella se ejecuta y el número de actividades que eso supone, y tiene mucho más que ver con la ampliación de derechos (culturales) y las 
posibilidades de la ciudadanía para ejercerlos de las más diversas formas (López Borbón, 2010).

Sin embargo - y aquí es donde entramos en la segunda parte de la afirmación inicial-, creer que porque existe esa dimensión inconmensurable de lo cultural no deben establecerse metas, objetivos e indicadores de resultados es un contrasentido absoluto con la gestión cultural. Es necesario, imperioso, pensar los proyectos de gestión cultural teniendo en cuenta estas dimensiones (Heras, 2008; Ander-Egg y Aguilar Ibáñez, 2000), especialmente cuando se habla de política pública en cultura, donde los gastos que se hacen deben responder a los ciudadanos. Sumado a esto, como fue destacado por Gonzalo Carámbula (2011), también es necesario porque persiste un abismo de comunicación entre los hacedores de políticas culturales y los decisores presupuestales; se hablan lenguajes distintos y por tanto es necesario tender puentes desde ambas orillas para enriquecer — no en términos económicos- las acciones que se llevan adelante.

\section{Notas finales}

Como se dijo a lo largo de este trabajo, no se ha pretendido hacer un análisis minucioso de los hechos que están detrás de los cambios y permanencias en las asignaciones y ejecuciones presupuestales en cultura, sino hacer una descripción analítica de esos cambios y permanencias. La diferencia entre una cosa y otra es clave a la hora de tomar este trabajo como cuestión cerrada o como una aproximación al problema que posibilite su instalación y preocupación, sobre todo por los involucrados en el sector cultural. El espíritu ha sido el segundo y, por tanto, las reflexiones que se plantean a continuación buscan bosquejar algunos fenómenos relacionados con lo hecho en políticas culturales, generar interrogantes hacia el futuro y nuevas líneas para investigar, vinculando la toma de decisiones en lo presupuestal con la gestión de las políticas públicas en cultura.

En relación con el armado de lo estrictamente presupuestal, sin lugar a dudas la perspectiva funcional es de lo más interesante, puesto que se propone trabajar por áreas programáticas y programas que buscan miradas transversales sobre las acciones de los organismos comprendidos dentro del Presupuesto Nacional. Sin embargo, para la tarea aquí planteada la perspectiva institucional ha sido de mayor utilidad en el entendido de que se buscó indagar sobre los organismos que tienen injerencia directa en el quehacer de las políticas públicas en cultura dentro del órgano rector en la materia, el Ministerio de Educación y Cultura.

En tal sentido, se mostró que el presupuesto real (o pasado a pesos constantes) asignado a la cultura solo pudo igualarse al que había en el año 2000 después de 14 
años, y luego de eso no pudo mantenerse y volvió a bajar. Además, el total asignado a estos organismos es sumamente marginal con relación al PIB uruguayo y al total del Presupuesto Nacional: tuvo sus mejores registros en el 2000, cuando representó el $0,285 \%$ del primero y el 1,22\% del segundo.

A lo largo de estas páginas se procuró mostrar algunos fenómenos que han sucedido en los últimos 20 años. Por una parte, que la inamovilidad de la institucionalidad cultural es solo aparente porque se han dado cambios significativos que se reflejan fuertemente en la forma en que se han asignado las partidas presupuestales. Posiblemente esto está fuertemente emparentado con el carácter aluvial de la institucionalidad de todo el MEC, ya destacada por Carámbula (2009 y 2011). Asimismo, se puede ver un cambio paulatino, aunque sumamente marcado, de desconcentración de la gestión en términos presupuestales desde la Dirección General de Secretaría -el organismo más cercano al/a ministro/a - hacia las otras UE. Si se sigue el razonamiento de Carámbula, sin duda el primero de estos fenómenos es un síntoma de falta de planificación sistémica y sistemática para emprender los asuntos culturales del país con una visión de largo plazo, mientras que el segundo es un muy buen síntoma en procura de formatos más saludables y autónomos de gestión menos vertical y jerárquica.

Otra cuestión que pudimos abordar es la confirmación de que el Sodre, por su histórica impronta polifuncional, ha sido la UE que mayores porcentajes de asignación ha tenido por fuera de la centralidad de la Dirección General de Secretaría. En tercero y cuarto lugar destacan el SECAN y la DNC respectivamente. A su vez, cada una de las UE trabajadas en este artículo muestra una mayor o menor flexibilidad en su asignación presupuestal según los tipos de gasto más coincidentes con las políticas públicas en cultura que desarrollan. Por ejemplo, Sodre, SECAN, DNC y Dirección General de Secretaría (particularmente $\mathrm{CMEC}$ ) son los que más gasto en funcionamiento tienen, lo cual es lógico por el tipo de acciones que despliegan. Lo mismo sucede en la Biblioteca Nacional y el Archivo General de la Nación, que son muy intensivos en personal, así como en la Comisión del Patrimonio Cultural de la Nación con el gasto en inversión.

Finalmente se mostraron, con el espíritu de promover que se indaguen con mayor precisión y pericia, los datos generales de la ejecución de esas UE en los últimos 20 años. La intención no era medir la eficiencia de esos organismos en la ejecución de sus políticas, sino plantear la necesidad de repensar ambas caras de la forma en que se gestiona la ejecución de los presupuestos en cultura. Es decir, poner sobre la mesa que las formas en que se presentan las metas, los objetivos y los indicadores -impuestas por el formato en que deben presentarse el Presupuesto Nacional y las Rendiciones de Cuentas - tal vez no sean las adecuadas para los hacedores de políticas públicas en cultura; así como proponer a los gestores culturales una adecuación de sentido, procurando traducir sus acciones al lenguaje de los decisores presupuestarios. Alfons Martinell decía: 
La gestión reclama una capacidad de definir objetivos y diseñar proyectos como eje y metodología de la acción. La gestión exige cierto gusto por la autonomía para decidir el curso de la acción y libertad para resolver problemas que emergen en la ejecución. La gestión se aproxima a una cierta creatividad en la búsqueda de alternativas e innovación con una gran sensibilidad de atención al exterior y a los procesos de su contexto. Y específicamente en el sector cultural, gestionar significa una sensibilidad de comprensión, análisis y respeto por los procesos sociales en los cuales la cultura mantiene sinergias importantes [...] La gestión de la cultura implica una valoración de los intangibles y asumir la gestión de lo opinable y subjetivo circulando entre la necesaria evaluación de sus resultados y la viabilidad de sus aspectos cuantitativos. (2001, p. 12)

\section{Bibliografía}

Ander-EGG, E. y Aguilar IbÁÑEz, M. J. (2000). Cómo elaborar un proyecto: Guía para diseñar proyectos sociales y culturales. (15. ${ }^{\mathrm{a}}$ ed.) Buenos Aires: Lumen. (Colección Política, Servicios y Trabajo Social).

BERRETTA, N. (2004). La asignación del gasto en la ejecución del presupuesto en Uruguay ¿resultado de las prioridades políticas definidas o de la casualidad? Montevideo: CINVE.

CARÁmbula, G. (2009). «Luz para la cara oculta de la Reforma del Estado». En G. RoBAINDA (coord.), Institucionalidad cultural (pp. 37-46). Montevideo: MEC.

CARÁmbula, G. (2011). «La institucionalidad cultural pública como problema». En F. Arocena (coord.), Regionalización cultural del Uruguay (pp. 295-355). Montevideo: Universidad de la República.

DE ToRRES, I. (2015). «Aportes para la historia de la radiodifusión como servicio público. El Sodre de Uruguay: una propuesta innovadora en el contexto hispanoamericano». Questión, 1(46), 97-110.

Decreto 52/11 (2011). Reglamentación del art. 52 Ley 18.719 sobre «contrato artístico» en sustitución del régimen «contrato de cachet». Recuperado de https://www.impo.com.uy/bases/decretos/52-2011.

Decreto 53/11 (2011). Reglamentación del art. 54 Ley 18.719 sobre contrato laboral con personas físicas por razones de necesidad expresamente justificadas. Recuperado de https://www.impo.com.uy/bases/decretos/53-2011.

Decreto 55/11 (2011). Reglamentación de los arts. 53 y 55 ley 18.719 sobre contrato temporal de derecho público. Recuperado de https://www.impo.com.uy/bases/ decretos/55-2011/1. 
Decreto 295/14 (2014). Reglamentación de la ley 19.037 relativa al marco legal de los museos. Recuperado de https://www.impo.com.uy/bases/decretos/295-2014.

HERAS, G. (2008). Gestión cultural y gestión de las artes escénicas en Iberoamérica. MéxiCO.

LAHERA PARADA, E. (2002). Introducción a las políticas públicas. Santiago de Chile: Fondo de Cultura Económica. (Breviarios del Fondo de Cultura Económica, 538)

Ley 18719 (2011). Presupuesto Nacional: Período 2010-2014. Recuperado de https://parlamento.gub.uy/documentosyleyes/leyes/ley/18719?width=800\&he ight $=600 \&$ hl=en_US1\&iframe=true\&rel=nofollow.

Ley 19.037 (2013). Museos. Recuperado de https://parlamento.gub.uy/documentosyleyes/ leyes/ley/19037?width=800\&height=600\&hl=en_US1\&iframe=true\&rel=nofollow.

Ley 19535 (2017). Rendición de cuentas y balance de ejecución presupuestal: Ejercicio 2016. Recuperado de: https://parlamento.gub.uy/documentosyleyes/leyes/ ley $/ 19535$ ?width=800\&height=600\&hl=en_US1\&iframe=true\&rel=nofollow.

LÓPEZ BORBóN, L. (2010). «Aproximaciones culturales a los márgenes urbanos en la época global: Una alternativa para la gestión incluyente en las grandes ciudades». En L. Álvarez, C. SÁnChEZ-Mejorada y C. SAN JuAn Victoria (coords.), La gestión incluyente en las grandes ciudades: estructura urbana, movilidad, seguridad y pluriculturalidad. México: UNAM.

MARTINELl, A. (2001). La gestión cultural: Singularidad profesional y perspectivas de futuro (recopilación de textos). Cátedra UESCO de Políticas Culturales y Cooperación.

NACIONES Unidas (1948). Declaración Universal de los Derechos Humanos. Recuperado de http://portal.unesco.org/es/ev.php-URL_ID=26053\&URL_DO=DO_TOPIC\&URL_ SECTION=201.html.

NACiones UnidAs (1966). Pacto Internacional de Derechos Sociales y Culturales. Recuperado de https://www.ohchr.org/SP/ProfessionalInterest/Pages/CESCR.aspx.

OPP (s/f.a). Portal de Transparencia Presupuestaria. Recuperado de https://transparenciapresupuestaria.opp.gub.uy/

OPP (s/f.b). «Presupuesto nacional». Portal de Transparencia Presupuestaria. Recuperado de https://transparenciapresupuestaria.opp.gub.uy/inicio/presupuestonacional.

Rausell, P. (dir.), Abeledo Sanchís, R., Carrasco Arroyo, S. y Martínez Tormo, J. (2007). Cultura: Estrategia para el desarrollo. Madrid: AECI.

Teixeira Coelho, J. (1997). Dicionário crítico de política cultural: Cultura e imaginário. São Paulo: Iluminuras.

UNESCO (1982). Declaración de México sobre las Políticas Culturales. Conferencia Mundial sobre las Políticas Culturales. Recuperado de http://www.culturalrights.net/ descargas/drets_culturals400.pdf. 